\title{
Electronic cigarettes and youth: a gateway that must be shut
}

\author{
Matthew B. Stanbrook MD PhD
}

See also page 794 and www.cmaj.ca/lookup/doi/10.1503/cmaj.151169

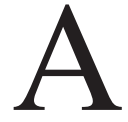
new study by Khoury and colleagues ${ }^{1}$ reports that $10 \%$ of a representative cohort of grade 9 students in Ontario had tried electronic cigarettes (e-cigarettes). This finding is likely an underestimate of rates across Canada: a 2013 study found substantial interprovincial variation in e-cigarette initiation among youth, with rates in Quebec double those in Ontario. ${ }^{2}$ Khoury and colleagues found that most were motivated to try e-cigarettes by their novelty and "coolness" - rarely did youth use e-cigarettes to quit smoking, arguably their only defensible use. E-cigarette use was highest among the most vulnerable youth, as reflected by poor health, high stress or low socioeconomic status. The study also confirmed that most were not substituting e-cigarettes for cigarettes: instead, the odds of e-cigarette use were 12-fold higher in youth who also smoked cigarettes (i.e., "dual users").

These findings add to mounting evidence associating use of e-cigarettes with tobacco use. A recent study involving US youth found that never-smokers in grades 11-12 who used e-cigarettes had sixfold higher odds of becoming cigarette smokers a year and a half later when they reached the legal age to purchase tobacco. ${ }^{3}$ Although this study, like previous ones, found that e-cigarette users were more likely to be identified on a validated measure as being inherently susceptible to smoking, susceptibility did not explain the association. Instead, lack of susceptibility magnified it: e-cigarette users not inherently susceptible to smoking had nearly 10 -fold higher odds of becoming smokers. These results are consistent with those of prior studies involving students in grades 9-10 and together leave little doubt that e-cigarette use can function as a gateway to tobacco smoking.

The spread of e-cigarettes to youth is not merely an innocent consequence of adolescents' natural inclination to experiment. It has been driven by aggressive marketing tactics by the industry - the same tactics that have long been used to promote tobacco to youth, including provocative ads and celebrity endorsements. Whereas Canada's official prohibition on nicotine-containing e-cigarettes has largely kept advertisements out of traditional media here, they are rampant on Internet and social media sites, where youth can be exposed to them readily. The typical fruit and candy flavourings of e-cigarette liquids are the number one reason they appeal to youth, ${ }^{4}$ and as with tobacco products, may mitigate deterrence from the initial harsh sensations that nicotine induces until dependence develops and fosters ongoing use.

E-cigarettes may renormalize smoking-like behaviours for youth and encourage such behaviours by introducing a popular factor - electronic technology - into an activity that public health efforts had succeeded in making increasingly unpopular among youth.

Fortunately, governments have begun to take action, albeit belatedly, to protect our youth from e-cigarettes. Although Khoury and colleagues collected data before the Ontario government's measures, Ontario and seven other provinces have now passed or tabled legislation that treats e-cigarettes similarly to tobacco products - including a prohibition on selling or supplying them to minors. Future studies will be needed to evaluate the effects of such legislation.

There is no good reason for youth - or any nonsmokers - to be using e-cigarettes. Nothing good can come of providing vulnerable individuals with a more appealing way to become addicted to nicotine. While we debate the question of whether e-cigarettes may enable harm reduction for smokers, we cannot ignore the simultaneous potential for harm creation, especially for youth. Mitigating this will require expansion of existing public health anti-tobacco programs to explicitly encompass e-cigarettes, including more active participation on social media. The federal government must prohibit flavourings in these products, because they entice youth and many are inherently harmful. Advertising restrictions currently in place for tobacco products must be explicitly extended to e-cigarettes. At an individual level, we all have an important role to play in engaging our youth in a conversation about the harms of e-cigarettes, lest we lose the progress against tobacco that we have worked so hard for decades to achieve.

\section{References}

1. Khoury M, Manlhiot C, Fan CPS, et al. Reported electronic cigarette use among adolescents in the Niagara region of Ontario. CMAJ 2016;188:794-800.

2. Czoli CD, Reid JL, Rynard VL, et al. E-cigarettes in Canada. Tobacco use in Canada: patterns and trends, 2015 edition, special supplement. Waterloo: Propel Centre for Population Health Impact, University of Waterloo; 2015. Available: www. tobaccoreport.ca/2015/TobaccoUseinCanada_2015_EcigaretteSupplement.pdf (accessed 2016 June 23)

3. Barrington-Trimis JL, Urman R, Berhane $\mathrm{K}$, et al. E-cigarettes and future cigarette use. Pediatrics 2016;138(1):e20160379.

4. Ambrose BK, Day HR, Rostron B, et al. Flavored tobacco product use among US youth aged 12-17 years, 2013-2014. JAMA 2015;314:1871-3.

Competing interests: See www.cmaj.ca/site/misc/cmaj_staff.xhtml.

Affiliation: Deputy Editor, CMAJ

Correspondence to: CMAJ editor, pubs@cmaj.ca

CMAJ 2016. DOI:10.1503/cmaj.160728 